\title{
Audiological Profile of Adult Persons with Auditory Neuropathy Spectrum Disorders
}

\author{
Pradeep Yuvaraj and Mannarukrishnaiah Jayaram \\ Department of Speech-Language Pathology \& Audiology, National Institute of Mental Health \& Neurosciences, Bangalore, India
}

$\begin{array}{ll}\text { Received } & \text { June 29, 2016 } \\ \text { Revised } & \text { July 20, 2016 } \\ \text { Accepted } & \text { August 3, 2016 }\end{array}$

Address for correspondence

Mannarukrishnaiah Jayaram, $\mathrm{PhD}$

Department of Speech-Language

Pathology \& Audiology,

National Institute of Mental Health \&

Neurosciences,

Hosur main road, Bangalore 560029,

India

Tel +918026995569

Fax +918026564830

E-mail drmjay16@gmail.com
Background and Objectives: The auditory profile of a large number of persons with late onset auditory neuropathy spectrum disorder (ANSD) is recently described in the Indian context. The purpose of study was 1) to profile data on routine audiological parameters, cortical evoked potentials, and temporal processing, 2) to analyze the benefit from hearing aids for persons with ANSD, and 3) to understand the association between benefit from hearing aids and auditory profile. Subjects and Methods: Thirty-eight adults with late onset ANSD and a matched group of 40 normally hearing adults participated in the study. Basic audiological tests, recording of cortical evoked potentials, and temporal processing tests were carried out on both groups of participant while only persons with ANSD were fitted with hearing aid. Results: Subjects in the two groups were significantly different on all the audiological parameters. ANSD group seemed to benefit from hearing aids variably. The mean amplitude of N2 was significantly different between normally-hearing participants and patients with ANSD. Conclusions: Residual temporal processing, particularly amplitude modulation detection seems to be associated with benefit from hearing aids in patients with ANSD.

J Audiol Otol 2016;20(3):158-167

KEY WORDS: Auditory neuropathy spectrum disorder - Gap detection threshold . Temporal resolution · Amplitude modulation detection · Hearing aid benefit.

\section{Introduction}

Individuals with auditory neuropathy spectrum disorder (ANSD) experience poor speech perception especially in the presence of back ground noise [1]. Starr, et al. [2] termed this form of hearing impairment as "auditory neuropathy". Absent auditory brainstem responses (ABRs), normal otoacoustic emissions (OAEs), and cochlear microphonics characterize this disorder [2]. ABRs, if present, are likely to be severely abnormal. In other words, persons with ANSD show normal outer hair cell functioning but abnormal auditory nerve conduction [3].

The site of lesion and the pathophysiology of ANSD are not yet completely understood. Cochlear amplification is preserved in these individuals, but discharges from the audi-

This is an Open Access article distributed under the terms of the Creative Commons Attribution Non-Commercial License (http://creativecommons.org/licenses/by-nc/3.0/) which permits unrestricted non-commercial use, distribution, and reproduction in any medium, provided the original work is properly cited. tory nerve are asynchronous. Some of the pathologies that lead to ANSD include damaged cochlear inner hair cells (IHC), abnormal IHC/auditory nerve synapse [4], disorder of spiral ganglion [5], reduced neuronal populations in the auditory brainstem [6], and demyelination of the auditory nerve [2]. Absent $8^{\text {th }}$ nerve [7], Charcot-Marie-Tooth Disease \& Mohr-Tranebjaerg syndrome [2,8], and mitochondrial diseases [9-11] are some of the disorders associated with over $30 \%$ of neuropathies.

\section{Audiological profile of children with ANSD}

Berlin, et al. [12] reported onset of ANSD before 4 years of age in more than $50 \%$ of all cases of ANSD and bilateral ANSD in $92 \%$ of the affected. In fact, Berlin, et al. [13] estimated that at least $4 \%$ of all children with permanent hearing loss may have ANSD. In a study of OAEs in 1,000 schoolgoing children with severe-to-profound hearing loss, Berlin, et al. [14] found that just $1 \%$ of these children showed robust unilateral or bilateral OAEs while another $10 \%$ showed small- 
er emissions in at least one ear. This perhaps indicates that a high proportion of children with ANSD may go unidentified. Similarly, Rance, et al. [15] reported evidence of ANSD in $11 \%$ of their children with permanent hearing deficit. Most of these children showed varying behavioral thresholds (normal to profound levels) and speech discrimination. Fifty percent of them did not even show awareness of speech input in both the unaided and aided conditions.

The underlying causes of ANSD make it difficult to estimate its prevalence [16]. In a study on the incidence and prevalence of ANSD in 5,199 infants with high risk for hearing loss, Rance, et al. [16] reported a prevalence of $0.23 \%$. They also reported a prevalence of $11 \%$ ANSD in 109 children with permanent moderate or higher hearing loss within the group. It is increasingly being realized that the incidence of ANSD is likely to go up in future with improved medical care available for children and infants with risk factors [16].

Kumar and Jayaram [17], in a register-based retrospective study, reported a prevalence of $0.28 \%$ of ANSD in all the hearing impaired and $0.54 \%$ in individuals with permanent hearing loss. ANSD was diagnosed on the basis of criteria of Starr, et al. [18] in this study. Kumar and Jayaram [17] reported a 'peaked' audiogram as the most common pure tone configuration, widely varying speech identification scores, an association between 'peaked' audiogram with better speech identification scores, and no association between speech identifi-cation scores and OAEs/ABRs in their participants. Fiftynine percent of the subjects in the study reported to have had the onset of the problem between 14 and 24 years signifying late onset of the problem in India. This observation was subsequently corroborated by Narne, et al. [19] who reported a mean onset age of 21.03 years (adolescence and adulthood) in $82 \%$ of their subjects while the remaining showed a childhood onset. This is in contrast to Berlin, et al. [12] who reported less than $10 \%$ of instances of onset in individuals between 16 to 18 years. While Kumar and Jayaram [17] reported a greater prevalence of the problem in males compared to females, Narne, et al. [19] reported the opposite of this (female to male ratio of 1.25:1). Narne, et al. [19] also reported a 'rising' pattern of pure tone audiogram and good speech identification in individuals in whom cortical potentials could be recorded. Furthermore, presence of cortical potentials was associated with increased benefit from hearing aids. Berlin, et al. [12] reported higher number of males (56\%) than females (44\%) affected with ANSD.

Previous research on the benefits from hearing aids to persons with ANSD has mostly focused on children and adults who had an early onset of the problem. Though there are some research as well as anecdotal reports on the benefit de- rived from hearing aids by adults $[12,13,20]$, not much is known about the age of onset of the problem in the subject population of these studies. Early or late onset of the problem seems to be an influencing factor in determining the degree to which persons with ANSD benefit from hearing aid amplification. Obviously, persons with late onset ANSD would have had a long period of normal hearing and auditory exposure and thus are likely to benefit more from hearing aids than others.

As part of a large project on the benefit from hearing aid to individuals with ANSD, data were obtained on parameters relating to cortical processing as well as temporal resolution (i.e., gap detection and amplitude modulation detection thresholds) in the present study. Though there is good information available on the auditory profile of individuals with ANSD, most of the data are on auditory parameters relating to type and nature of hearing loss, speech identification and comorbid features of ANSD. Therefore, the purpose of the present report was to present data not only on the basic profile of hearing (e.g., pure tone, speech identification, OAE's, and ABR), but also on measures of suprathreshold perception (or temporal resolution), cortical processing of auditory information and on the benefit from hearing aid amplification in a large sample of persons with late onset ANSD.

\section{Subjects and Methods}

\section{Subjects}

The participants were selected based on the criteria of Starr, et al. [18] for identification of ANSD (preserved cochlear amplification, normal OAEs, abnormal or absent ABRs and no acoustic reflexes). A clinical examination showed no neurological or otological disorder in any of the participants.

Detailed information was elicited on the age of onset of the problem, characteristics of the hearing problem and history of intervention, if any. In the final analysis, thirty-eight individuals, aged between 16 and 30 years (mean age $=22.38$ years) and with a confirmed diagnosis of ANSD participated in the study. Participants included both males $(n=25)$ and females $(n=13)$. Age of onset of the problem ranged from 9 to 29 years (mean age of onset $=16.08$ years). However, the reliability of this information is questionable as there are no medical records to back the assertion. None of the participants had any family history of the problem. Six persons reported hyperbilirubinemia (report of having jaundice), but no information relating to etiology of the problem could be ascertained from others. Participants with ANSD in the present study showed poor speech identification in both quiet $(n=37)$ and in noise $(n=38)$. Twenty-nine persons reported gradual 
onset of the problem while it was sudden in the remaining. All the participants spoke Kannada-a Dravidian languagespoken by about 70 million people predominantly in the South Indian state of Karnataka.

Forty normally hearing individuals matched for age, gender, education, socioeconomic status and language-spoken with those in the experimental group served as controls. A structured interview confirmed that persons in the normallyhearing (control) group had no difficulty in hearing/understanding speech in daily listening conditions. A routine clinical examination ruled out any neurological or otological problem. The normally-hearing participants underwent the same tests as participants with ANSD for inclusion into the study. Normally-hearing participants showed pure tone thresholds of less than $20 \mathrm{~dB}$ HL (ISO 389) (3-frequency average $-500,1,000$ and $2,000 \mathrm{~Hz}$ ), speech identification scores $>95 \%$ at $40 \mathrm{~dB}$ SL (ref: pure tone average), 'A' type tympanogram with reflexes present at normal sensation levels, identifiable ABRs (waves I, III, and V) at normal latencies, and normal TEOAEs. After recruitment into the study, the normal hearing participants underwent all the tests as subjects in the ANSD group except hearing aid fitting. All participants were recruited into the study after approval from the Institute Ethics Committee of the National Institute of Mental Health \& Neurosciences, Bangalore, India (No. NIMH/65th IEC/ 2009, dated 19.3.2009: Item 11.01).

\section{Basic audiologic tests}

\section{Pure tone and speech audiometry}

Air conduction and bone conduction pure tone thresholds for octave frequencies were obtained using a calibrated clinical audiometer (GSI-61; Grason Stadler, Eden Prairie, MA, USA). Modified version of Hughson and Westlake procedure was followed with a presentation of $5 \mathrm{~dB}$ step size. Pulsed tone was employed to test participants reporting tinnitus. All hearing tests were conducted in a sound treated room built as per ANSI (1991) standards for noise levels [21].

\section{Speech identification}

Testing was done with live voice presentation of phonetically balanced monosyllables at $40 \mathrm{~dB}$ SL. Speech was presented from a calibrated audiometer (GSI-61) through headphones (TDH39; Telephonics, Huntington, NY, USA). The intensity of speech output was regulated through audiometer. The participants were asked to repeat the words they heard and the responses were audio-recorded for later verification.

\section{Immittance evaluation}

Immittance evaluation for $226 \mathrm{~Hz}$ probe tone was carried out with a middle ear analyzer (GSI Tympstar, Grason Stadler). Ipsilateral and contralateral acoustic reflexes were recorded at $500,1,000$, and 2,000 Hz. Participants were instructed not to move or swallow during the procedure.

\section{Auditory brainstem responses}

ABRs were recorded (IHS Smart EP; Intelligent Hearing Systems, Miami, FL, USA) using a standard protocol (active electrode- forehead; reference electrode-ipsilateral mastoid; ground electrode-contralateral mastoid, bandpass filter between 100 and 3,000 Hz; condensation as well as rarefaction click stimuli at a rate of 11.1 clicks per second through insert earphones-ER.3A-Etymotic Research, Elk Grove Village, USA; electrode impedance $<5 \mathrm{kOhms}$ ). Two traces for rarefaction and one for condensation polarity were recorded to check on the nature of waves obtained. Reversal of the waves for condensation indicated presence of cochlear microphonics. The participants were instructed to rest and refrain from any activity during the procedure to avoid artifacts.

\section{Otoacoustic emissions}

TEOAEs were recorded (Echoport ILO 292-II; Otodynamics Ltd., Herts, UK) for clicks at $80 \mathrm{~dB}$ SPL pe (peak equivalent SPL). Waveform reproducibility of $90 \%$ or more and a signal-to-noise ratio, of $\mathrm{dB}$ or more were prerequisites for a valid emission. The position of the probe during recording was not altered. The default stimulation level through the adult probe was 0.3 Pascal ( $80 \mathrm{~dB}$ SPL). The stimulus was repeated at intervals of 20 milliseconds which allowed enough time for acoustic emissions to return from the farthest part of the cochlea.

\section{Temporal resolution measurement}

\section{Gap in noise test}

Tests for temporal resolution were through an mlp (maximum likelihood procedure) toolbox in Matlab (R2008b; Mathworks, Natick MA, USA) [22]. The ability of the participants to detect a period of silence in the center of a 750 milliseconds band pass noise was tested. Duration of the gap varied depending on the response of the participants on mlp. The noise had 0.5 milliseconds cosine ramp at the beginning and end of the gap. In two-alternate force choice tasks, there would be two stimuli-a standard and a variable one. The standard stimulus is a 750 milliseconds broadband noise with no gap while the variable stimulus had a gap of varying duration. The order of presentation of standard and variable stimuli 
was random.

The MATLAB-generated stimuli were presented to the participants through a clinical audiometer (GSI-61). In each trial, a standard stimulus (with no gap) and a variable stimulus (two bursts of white noise separated by an interval) were presented. The task of the participants was to identify the sequence that had a gap. There are reports suggesting that individuals with auditory dys-synchrony have severe problems in perceiving silent gaps of even 20 to 30 milliseconds [23]. Therefore, the test started with presentation of stimulus that had a silent interval of 64 milliseconds. Thereafter, the magnitude of the duration of gap changed according to the response of the participant (as provided for in the protocol of maximum likelihood procedure of MATLAB) [24]. Participants were given 10 practice presentations before the commencement of the test. Four sequences of 30 stimuli each were presented. The MATLAB protocol provides for computing the average gap detection threshold in each sequence, and then across the four sequences. The final gap detection threshold is the average gap detection for 120 presentations in each ear.

\section{Amplitude modulation detection test}

A measure of the extent of amplitude variation about an un-modulated carrier is the modulation index ( $\mathrm{M}=\mathrm{RMS}$ value of modulating signal/RMS value of un-modulated signal). Modulation index is known as modulation depth when expressed as a percentage. Thus, a modulation index of 0.5 can be expressed as a modulation depth of $50 \%$. Modulation index varies from 0 to 1 where ' 0 ' stands for 'no modulation' and ' 1 ' for ' $100 \%$ modulations'. The modulation detection threshold in $\mathrm{dB}$ is equal to $20 \log _{10}(\mathrm{M})$.

Participants were tested for their ability to detect amplitude modulations in a signal. All amplitude-modulated stimuli were generated on the MATLAB platform. Gaussian noise of 500 milliseconds duration was sinusoidally amplitude modulated at 8,20 , and $60 \mathrm{~Hz}$. Depth of the modulated signal changed depending upon the response of the participants up to $80 \%$ criterion level (as provided for in the MATLAB platform).

The MATLAB-generated stimuli were presented through a clinical audiometer (GSI-61). Each presentation consisted of two stimuli, one after the other: one of these stimuli was modulated. For example, one stimulus was a standard noise and other modulated (variable depth). The task before the participants was to identify the modulated stimulus. Four sequences, each with 30 stimuli, were presented. At the end of 30 presentations, a modulation detection threshold was obtained. The final modulation detection threshold is the aver- age of the detection thresholds of all the four sequences (120 presentations for each ear). A similar procedure was used to obtain modulation detection thresholds for signals with modulations of 8,20 , and $60 \mathrm{~Hz}$. The order of presentation of stimuli was kept different for the two ears to avoid adaptation effect.

\section{Cortical evoked potentials}

Long latency responses (LLR) were recorded for both clicks and a natural CV syllable/ta/ spoken by an adult female speaker. Speech stimulus was recorded at a sampling frequency of $16 \mathrm{kHz}$ with 16-bit digitization. The duration of the stimulus was limited to 100 milliseconds by deleting the steady state of the vowel at the end of the syllable [25]. The pre-stimulus electrical activity was subtracted from the response waveforms to correct the waveforms for baseline EEG activity. Stimuli were presented at $40 \mathrm{~dB}$ SL (ref: PTA $500,1,000$, and $2,000 \mathrm{~Hz}$ ) to ensure comfortable level of hearing. Standard recording protocol was used (bandpass filter between 1 to $30 \mathrm{~Hz}$; rate of presentation at 1.1 clicks per second through ER.3A insert earphones). $\mathrm{Cz}$ was the active electrode with reference electrode at ipsi lateral mastoid and ground electrode at Fz. Data was acquired only after ensuring $<5 \mathrm{k} \Omega$ impedance at all electrode sites.

Prior to analysis of individual waveforms, grand averages of late latency responses (LLRs) (separately for speech and clicks; and for right and left ear) were generated. Grand average waveforms were obtained by averaging individual recordings of 36 individuals with ANSD. The experimenter and another experienced audiologist identified all the waves and made all the measurements. Only those waves and measures on which there was $100 \%$ agreement between the two judges were considered for analysis.

\section{Hearing aid fitment}

Speech identification testing in quiet was carried out in a sound treated room. The audio-recorded speech stimuli were presented at a comfortable level from an audiometer through a pair of loud speakers kept at a distance of 1 meter and $0^{\circ}$ azimuth. Speech testing in quiet was binaural.

Hearing aids were fitted binaurally. Hearing aids were programmed individually for each participant with auditory dyssynchrony depending upon the configuration of his pure tone audiogram. Generally, a 4 to 9 channel high quality hearing aid with wide dynamic range and low gain was selected. The hearing aids were programmed using HI-PRO (Otometrics, Taastrup, Denmark). Aided speech identification testing procedure (with binaural hearing aids) was similar to that for unaided speech identification testing. 
Aided as well as unaided speech identification testing was carried out by presenting words from a list of 500 most familiar words in written Pradeep [26]. Each of these 'most familiar words' occurred 50 to 55 times in 100,000 words of randomly selected printed text in Kannada. The longest words in these lists were four syllables long. 25 lists, each with 20 randomly selected words, were prepared for convenience. The word lists were 'prepared' through randomization and the assumption was that phoneme distribution in different word lists will be in the same proportion as found in written published text. It is possible that phoneme representation across word lists (for example, word-initial phoneme) was not perfectly uniform across the lists. Each participant was tested on four lists of 20 words each (two lists in the aided and two lists in the unaided condition). Though the word lists were selected randomly for each participant, care was taken to ensure that the lists were different for aided and unaided testing for each participant. Aided speech identification scores were obtained only from participants with auditory dys-synchrony. The difference between the aided and unaided speech identification score was considered the hearing aid benefit score.

\section{Results}

Some of the auditory and non-auditory characteristics of participants with ANSD of this study are given in Table 1 .

Non-parametric statistical tests (i.e., Mann Whitney U test and the Wilcoxon Signed Rank test) were used for testing the significance of difference of means instead of ANOVA tests (parametric) because the high standard deviation in respect of all parameters for participants with auditory dys-synchrony may violate the assumption of normal distribution. Wilcoxon Signed rank test showed no significant difference between the mean scores of the two ears for any parameter either for participants with ANSD or normally-hearing $(p<0.01)$. Therefore, the average of the scores of the two ears for each of these parameters was considered in all statistical analysis.

The normally-hearing and ANSD groups were compared for significance of difference of means for a number of audiological parameters and the results are shown in Table 2. Results showed that the normally-hearing were significantly different from participants with ANSD on all audiological parameters tested.

\section{Late latency responses}

A comparison of mean latency and amplitude of click evoked LLRs between normally-hearing and ANSD groups (Table 3 ) showed that the two groups were not significantly different $(p>0.01)$ with reference to either latency or amplitude of peaks. Results of a similar analysis for speech evoked LLRs are shown in Table 4. Man Whitney test showed that only the mean amplitude of N2 was significantly different

Table 1. Demographic characteristics of individuals with ANSD

\begin{tabular}{lc}
\hline \multicolumn{1}{c}{ Characteristics } & ANSD \\
\hline Male - female ratio & $1.92: 1$ \\
Mean age of onset & 16.08 years \\
Difficulty in speech identification in noise & $100 \%$ \\
Difficulty in speech identification in quiet & $97.36 \%$ \\
Difficulty in identifying non-verbal sound & $5.27 \%$ \\
Tinnitus & $26.31 \%$ \\
Progressive nature of the problem & $76.32 \%$ \\
Onset & \\
Gradual & $76.32 \%$ \\
Sudden & $23.68 \%$ \\
Intolerance to loud sounds & $2.63 \%$ \\
Hearing aid usage & $5.27 \%$ \\
Difficulty conversing in a group & $100 \%$ \\
Difficulty understanding speech from TV/radio & $100 \%$ \\
Anxiety while speaking over phone & $86.84 \%$ \\
History of neonatal jaundice & $15.79 \%$ \\
Giddiness & $15.79 \%$ \\
Change in voice & $10.53 \%$ \\
\hline ANSD: &
\end{tabular}

ANSD: auditory neuropathy spectrum disorder

Table 2. Mean, SD and significance of difference of mean scores between the normally- hearing and persons wit ANSD

\begin{tabular}{|c|c|c|c|c|c|c|}
\hline \multirow{2}{*}{ Tests } & \multicolumn{2}{|c|}{ Normally hearing } & \multicolumn{2}{|c|}{ ANSD group } & \multirow{2}{*}{ Mann Whitney U } & \multirow{2}{*}{$p$} \\
\hline & Mean & SD & Mean & SD & & \\
\hline PTA & 10.93 & 1.94 & 35.67 & 12.84 & 45.00 & $<0.001$ \\
\hline SIS & 98.93 & 1.48 & 60.19 & 24.98 & 0.00 & $<0.001$ \\
\hline TEOAE & 19.46 & 1.48 & 17.51 & 4.56 & 452.50 & $<0.001$ \\
\hline GDT & 3.07 & 0.22 & 16.38 & 12.25 & 0.00 & $<0.001$ \\
\hline TMTF $8 \mathrm{~Hz}$ & -12.76 & 3.13 & -8.54 & 2.68 & 252.00 & $<0.001$ \\
\hline TMTF $20 \mathrm{~Hz}$ & -17.26 & 2.20 & -7.45 & 2.52 & 0.00 & $<0.001$ \\
\hline TMTF $60 \mathrm{~Hz}$ & -12.08 & 1.90 & -6.26 & 2.09 & 26.00 & $<0.001$ \\
\hline
\end{tabular}

SD: standard deviation, ANSD: auditory neuropathy spectrum disorder, PTA: pure tone average, SIS: speech identification scores, TEOAE: transient otoacoustic emissions, GDT: gap detection thresholds, TMTF: temporal modulation transfer function 
$(p<0.01)$ between the two groups.

\section{Benefit from hearing aid amplification}

Thirty of the thirty eight participants showed improved speech identification with hearing aids. The improvement ranged from 5 to 50 percent. Therefore, the midpoint of this range $(22.5 \%)$ was considered to divide the group into those who benefitted (Group 1) and those who did not (Group 2). Accordingly, there were 12 persons in Group 1 and 26 in Group 2. An analysis was carried out to see if the two groups were significantly different with respect to measures of temporal resolution (gap detection and amplitude modulation detection) and the results are given in Table 5. Results of Mann Whitney test showed that the group which benefitted from hearing aids showed significantly better values of gap detection and amplitude modulation detection thresholds than the group which did not benefit from hearing aids $(p<0.01)$.

A correlational analysis was carried out between different auditory parameters and benefit from hearing aids. The re-

Table 5. Mean, SD and significance of difference of mean scores between the two groups who variably benefitted from hearing aids

\begin{tabular}{|c|c|c|c|c|c|}
\hline & \multicolumn{2}{|c|}{ Groupl $(n=12)$} & \multicolumn{2}{|c|}{ Group $2(n=26)$} & \multirow{2}{*}{$P$} \\
\hline & Mean & SD & Mean & SD & \\
\hline PTA (dB) & 43.88 & 8.98 & 31.92 & 12.70 & $<0.01$ \\
\hline SIS & 66.45 & 9.62 & 57.30 & 29.25 & 0.889 \\
\hline GDT & 11.42 & 1.78 & 18.66 & 14.26 & $<0.01$ \\
\hline TMTF $8 \mathrm{~Hz}$ & -11.11 & 1.22 & -7.36 & 2.32 & $<0.01$ \\
\hline TMTF $20 \mathrm{~Hz}$ & -9.77 & 1.02 & -6.38 & 2.27 & $<0.01$ \\
\hline TMTF $60 \mathrm{~Hz}$ & -8.19 & 1.46 & -5.37 & 1.71 & $<0.01$ \\
\hline
\end{tabular}

SD: standard deviation, PTA: pure tone average, SIS: speech identification scores, GDT: gap detection thresholds, TMTF: temporal modulation transfer function

Table 3. Mean, SD and the significance of difference of mean latency and amplitude of click evoked LLRs between normal hearing and ANSD groups

\begin{tabular}{|c|c|c|c|c|c|c|}
\hline & \multicolumn{2}{|c|}{ Normally hearing } & \multicolumn{2}{|c|}{ ANSD group } & \multirow{2}{*}{ Mann Whitney U } & \multirow{2}{*}{$p$} \\
\hline & Mean & SD & Mean & SD & & \\
\hline \multicolumn{7}{|l|}{$\mathrm{Pl}$} \\
\hline Latency & 67.03 & 5.73 & 64.81 & 15.91 & 742.00 & 0.85 \\
\hline Amplitude & 2.37 & 0.42 & 2.25 & 0.60 & 732.00 & 0.77 \\
\hline \multicolumn{7}{|l|}{$\mathrm{N} 1$} \\
\hline Latency & 116.36 & 8.89 & 110.58 & 27.21 & 660.50 & 0.32 \\
\hline Amplitude & -0.45 & 0.08 & -0.42 & 0.17 & 640.00 & 0.23 \\
\hline \multicolumn{7}{|l|}{ P2 } \\
\hline Latency & 146.03 & 9.09 & 143.43 & 34.53 & 563.00 & 0.04 \\
\hline Amplitude & 2.81 & 0.29 & 2.67 & 0.70 & 730.50 & 0.76 \\
\hline \multicolumn{7}{|l|}{ N2 } \\
\hline Latency & 202.52 & 10.67 & 190.97 & 46.25 & 672.00 & 0.37 \\
\hline Amplitude & -1.64 & 0.18 & -1.47 & 0.49 & 583.00 & 0.07 \\
\hline
\end{tabular}

SD: standard deviation, LLRs: late latency responses, ANSD: auditory neuropathy spectrum disorder

Table 4. Mean, SD and the significance of difference of mean latency and amplitude of speech evoked LLRs between normal hearing and ANSD groups

\begin{tabular}{|c|c|c|c|c|c|c|}
\hline & \multicolumn{2}{|c|}{ Normally hearing } & \multicolumn{2}{|c|}{ ANSD group } & \multirow{2}{*}{ Mann Whitney U } & \multirow{2}{*}{$p$} \\
\hline & Mean & SD & Mean & SD & & \\
\hline \multicolumn{7}{|l|}{$\mathrm{Pl}$} \\
\hline Latency & 66.86 & 5.73 & 65.07 & 15.96 & 722.00 & 0.70 \\
\hline Amplitude & 2.39 & 0.47 & 2.25 & 0.63 & 684.50 & 0.45 \\
\hline \multicolumn{7}{|l|}{$\mathrm{N} 1$} \\
\hline Latency & 116.36 & 8.94 & 110.20 & 27.54 & 691.00 & 0.49 \\
\hline Amplitude & -0.45 & 0.09 & -0.37 & 0.18 & 530.50 & 0.02 \\
\hline \multicolumn{7}{|l|}{ P2 } \\
\hline Latency & 146.04 & 9.12 & 143.46 & 34.61 & 554.00 & 0.03 \\
\hline Amplitude & 2.82 & 0.28 & 2.64 & 0.76 & 671.50 & 0.37 \\
\hline \multicolumn{7}{|l|}{ N2 } \\
\hline Latency & 202.51 & 10.71 & 189.88 & 46.13 & 633.50 & 0.20 \\
\hline Amplitude & -1.63 & 0.18 & -1.40 & 0.47 & 464.00 & $<0.01$ \\
\hline
\end{tabular}

LLRs: late latency responses, ANSD: auditory neuropathy spectrum disorder, SD: standard deviation 
Table 6. Correlation between PTA, SIS in quiet, GDT and temporal modulation transfer function (amplitude modulation detection threshold-TMTF) thresholds (at 8,20 and $60 \mathrm{~Hz}$ ), and benefit from hearing aid score

\begin{tabular}{lccccccc}
\hline & PTA & \multicolumn{1}{c}{ SIS } & \multicolumn{1}{c}{ GDT } & \multicolumn{1}{c}{ TMTF 8 Hz } & \multicolumn{1}{c}{ TMTF 20 Hz } & TMTF 60 Hz & Benefit score \\
\hline PTA & & $-0.27(0.09)$ & $0.12(0.44)$ & $-0.02(0.90)$ & $-0.03(0.81)$ & $-0.11(0.49)$ & $0.42(0.008)$ \\
SIS & $-0.27(0.09)$ & & $-0.40(0.01)$ & $-0.42(0.008)$ & $-0.38(0.01)$ & $-0.25(0.12)$ & $-0.04(0.77)$ \\
GDT & $0.12(0.44)$ & $-0.40(0.01)$ & & $0.73(<0.001)$ & $0.71(<0.001)$ & $0.69(<0.001)$ & $-0.35(0.02)$ \\
TMTF $8 \mathrm{~Hz}$ & $-0.02(0.90)$ & $-0.42(0.008)$ & $0.73(<0.001)$ & & $0.94(<0.001)$ & $0.89(<0.001)$ & $-0.48(0.002)$ \\
TMTF 20 Hz & $-0.03(0.81)$ & $-0.38(0.01)$ & $0.71(<0.001)$ & $0.94(<0.001)$ & & $0.93(<0.001)$ & $-0.41(0.01)$ \\
TMTF 60 Hz & $-0.11(0.49)$ & $-0.25(0.12)$ & $0.69(<0.001)$ & $0.89(<0.001)$ & $0.93(<0.001)$ & & $-0.47(0.003)$ \\
\hline PTA $:$ PUF
\end{tabular}

PTA: pure tone average, SIS: speech identification scores, GDT: gap detection thresholds

sults are shown in Table 6 . There was a negative correlation between 3-frequency PTA and benefit from hearing aids. On the other hand, TMTF $8 \mathrm{~Hz}$, TMTF $20 \mathrm{~Hz}$ and TMTF $60 \mathrm{~Hz}$ detection thresholds were positively correlated with benefit from hearing aids.

\section{Discussion}

\section{Non-auditory characteristics}

In the final analysis, 38 individuals (25 males and 13 females), in the age range between 16 and 30 years (mean age $=$ 22.38 years) and with confirmed diagnosis of ANSD formed the clinical group. Majority of the participants of this study reported a late onset of ANSD (average age of onset of the problem was 16.08 years). However, as there were no medical records maintained for any of these participants, the validity of information given by the participants is always debatable and the results of this study should be interpreted considering this fact.

Speech identification was affected in noise $(n=38)$ as well as in quiet $(n=37) .36$ of the 38 participants reported no difficulty in perceiving non-verbal sounds. The etiology of ANSD could not be ascertained in any of these subjects because of lack of medical records, but 6 participants (15.78\%) reported history of hyperbilirubinemia. Berlin, et al. [12] reported a higher percentage $(48.37 \%)$ of hyperbilirubinemia in their subjects with ANSD.

\section{Auditory characteristics}

Pure tone audiometry indicated that participants with auditory dys-synchrony had normal to moderate hearing loss. This observation, in itself, is not significant because only subjects with mild to moderate hearing loss were recruited into the study. All the participants showed symmetric hearing loss. The results on pure tone thresholds demonstrate the well reported observation that speech identification scores are disproportionate to the degree of hearing loss in persons with ANSD. Most of the participants of this study with ANSD had hearing loss predominantly in the low frequency region.
The reason for low frequency hearing loss in persons with ANSD is well understood $[3,27]$.

Speech identification in individuals with ANSD has been well documented in the literature. Speech identification score disproportionate to the degree of hearing loss [2] is considered a cardinal feature of persons with ANSD. The results of the present study provide additional evidence to this observation.

A comparison of mean speech identification scores of those who benefitted from hearing aids with those who did not showed no significant difference in the mean scores between the two groups (Table 5). The highly scattered scores in each group (Group 1; 45 to $82.5 \%$ and Group 2; 0 to $90 \%$ ) may be the reason for this lack of significant difference. This result implies that benefit from hearing aid and speech identification scores are not associated.

Synchronous firing of auditory nerve fibers is important for extracting complex acoustic features such as spectral peaks and waveform envelopes for speech recognition. The inability to follow temporal fluctuations is an important cause for poor speech perception in individuals with ANSD [28]. The participants with ANSD of the present study showed poor ability to detect amplitude modulations in a steady state signal (discussed under the section on amplitude modulation detection threshold).

Presence of OAEs in individuals with ANSD has been extensively reported $[29,30]$. The mean amplitude of transient otoacoustic emissions in persons with ANSD was $17.51 \mathrm{~dB}$ in the present study. Starr, et al. [18] and Michalewski, et al. [31] reported that some individuals with ANSD may lose OAE's over time. There may be some supporting evidence for this observation in the present study in that OAEs were absent in one of the participants of the study with ANSD (who had the problem of ANSD for 21 years at the time of testing). OAEs were reduced in amplitude (9.6 and $8.75 \mathrm{~dB}$ ) in two other participants of the study. Whether reduced amplitude would continue to eventually result in the loss of OAE's over time needs to be investigated. 


\section{Temporal resolution}

\section{Gap detection}

Normally hearing persons can perceive silent periods of $<5$ milliseconds while individuals with ANSD usually require gaps of 20 milliseconds or more before they become aware of the change $[2,27,32,33]$. Gap detection thresholds give information on an individual's ability to process temporal information in the speech signal. Results on gap detection thresholds of persons with ANSD in the present study (range: 11 to 64.4 milliseconds) indicate that temporal processing is severely impaired in this clinical population which has been reported by many others in the past [27,34]. Dys-synchronized auditory nerve activity impairs the ability to perceive temporal cues like voice onset time, burst duration, etc. An inability or a reduced ability to detect brief gaps in the speech signal affects the perception of vowel features such as third formant onset frequency [1] and discrimination of place of articulation of consonants (which is based upon subtle differences in voice onset time) [35].

\section{Amplitude modulation detection threshold}

Temporal resolution was assessed by measuring the subjects' ability to detect rapid fluctuations in the amplitude of the signal (at different modulation rates). Previous reports on amplitude modulation detection thresholds in individuals with ANSD are variable, but have generally reported a decreased or impaired capacity to detect fast amplitude changes over time $[27,34,36]$.

The present study investigated the modulation detection thresholds for sinusoidally amplitude modulated Gaussian noise at 8, 20, and $60 \mathrm{~Hz}$. Results of the present study showed that the detection thresholds improved with decrease in modulation frequency (better thresholds for $8 \mathrm{~Hz}$ modulations than for 20 and $60 \mathrm{~Hz}$ ). Similar results have been reported earlier [7] and the findings have been well explained [17].

Low frequency modulations are decoded at the cortical level while the high frequency modulations are decoded at the level of the lower brain stem [36]. Since the site of lesion in individuals with ANSD is supposedly the IHC-auditory nerve synapse or the auditory nerve itself, the dys-synchrony at this level affects perception of high frequency modulations more than perception of low frequency modulations.

\section{Cortical evoked potentials}

LLRs for clicks and speech could be recorded in 36 of the 38 participants of the present study even though none of them showed ABRs. Generation of cortical evoked potentials does not depend upon neural synchrony to the same ex- tent as ABRs [37].

The results of the present study showed that the amplitude of N2 was significantly different between the normally-hearing and the auditory dys-synchrony group. Oates, et al. [38] reported 1) decreased N2 amplitude in children and adults with sensori-neural hearing loss and furthermore, 2) that the decrease in N2 amplitude was dependent on duration of hearing loss. Extrapolating these findings, Yuvaraj and Mannarukrishnaiah [39] have explained that the lack of, or low representation of acoustic features of sound at the cortical level over a period may be the reason for lower N2 amplitude in individuals with auditory dys-synchrony. Furthermore, the representation or non-representation of sound at the cortical level may not, in itself, be important because N2 amplitude is decreased even in sensori-neural hearing loss. It is perhaps the poor representation of the acoustic features of sound stimulus that may be responsible for lower amplitude of N2 in persons with ANSD [39].

\section{Hearing aid benefit}

Previous researches $[2,28]$ have generally reported poor benefit from hearing aids for individuals with ANSD. There was a statistically significant difference between unaided $($ mean $=47.63 \%)$ and aided $($ mean $=61.84 \%)$ speech identification scores for participants in the ANSD group. This result is in partial agreement with past research [15,31]. It is reported that individuals with ANSD do benefit from hearing aids with low gain and wide dynamic range [15,16,31]. It is also said that not all individuals with ANSD have completely lost temporal processing abilities [27,36]. Therefore, it is our assumption that a subgroup of individuals with ANSD with residual temporal processing ability may benefit from hearing aids.

In general, 30 of the 38 persons with ANSD benefitted from hearing aid amplification. Twelve of these showed greater than 22.5 percent improvement over the unaided score. This suggests a need for revisiting the issue of benefit from hearing aid for individuals with ANSD. Correlation analysis indicated that benefit from hearing aids is associated with higher amplitude detection threshold which shows the importance of temporal processing (resolution) in the perception of speech. It can be contended that those individuals with ANSD who had better detection thresholds for slow modulations $(8 \mathrm{~Hz})$ identify speech better even without hearing aids. The reasons for higher speech identification score with hearing aids in such participants can only be speculated.

There is evidence to suggest that processing of speech in hearing aids results in temporal distortion of the signal. Spectral contrast and signal overshoot are the two major consequences of unfavorable temporal behavior in hearing aids 
with wide dynamic range compression systems which adversely affect speech identification [40]. Speech comprises of three main temporal features of envelop, periodicity and fine structure [41], and temporal distortion refers to modification of any of these temporal features of speech. Temporal distortion of speech while being processed in a digital hearing aid may affect both clarity and naturalness of sound. It is speculated here that those individuals with ANSD who have better residual temporal processing abilities are able to overcome the distortion of temporal parameters in hearing aid processed speech and thus, benefit from hearing aid amplification.

Gap detection threshold (another measure of temporal resolution) was not significantly correlated with benefit from hearing aids. Though the participants of this study had a mean GDT of 16.38 milliseconds, they had a mean unaided speech identification score of $47.63 \%(n=38)$. It has been reported [42] that within-channel gap detection tests (using a signal that is identical both preceding and following a gap) like GIN test may not accurately represent the type of temporal resolution and acuity that is important for speech perception. As such, gap detection threshold may not be a good predictor of the benefit derived from hearing aids. On the other hand, the mean gap detection thresholds were significantly different between the group of persons with ANSD who benefitted from hearing aids and those who did not (Table 5). Participants in Group 1 had better gap detection thresholds and benefitted more from hearing aids. In contrast, participants in Group 2 had poorer gap detection thresholds and did not benefit much from hearing aids. The discrepancy between the results of group comparison (Table 5) and correlational analysis (Table 6) indicates the need to revisit the relationship between gap detection threshold and speech perception.

\section{Pure tone thresholds and benefit from hearing aids}

The two groups based on benefit from hearing aid were significantly different with respect to mean PTA. Those who benefitted from hearing aid (Group 1) had significantly poorer thresholds than those who did not benefit (Group 2). The correlation analysis also supports this observation (Table 6) (column 7, row 2). This perhaps reflects on the role of hearing aids in augmenting speech detection in individuals with ANSD. In persons with poorer hearing thresholds, augmentation of the signal through hearing aids may help in the detection of the signal. Javel [43] has reported that the afferent nerve fibers show increased phase locking and synchronous discharge at higher sensation levels. It has been speculated [19] that higher sound levels may aid perception by improving neural synchrony. This may explain the results of the present study on the relationship between poorer PTA and higher benefit from hearing aids. Future research is warranted not only to check the reliability of this observation, but also to explain the phenomenon if it is found to be reliable observation.

The audiological profile of a large sample of persons with late onset ANSD is described. The profile includes data not only on routine auditory parameters, but also on cortical evoked potentials and supra threshold processing (measures of temporal resolution). In addition, the importance of this auditory data in understanding the benefit derived from hearing aids by persons with ANSD is discussed. The importance of residual temporal processing abilities for deriving benefit from hearing aids is highlighted as also the relationship between pure tone hearing loss and benefit from hearing aids.

\section{Conflicts of interest}

The authors have no financial conflicts of interest.

\section{REFERENCES}

1) Kraus N, Bradlow AR, Cheatham MA, Cunningham J, King CD, Koch DB, et al. Consequences of neural asynchrony: a case of auditory neuropathy. J Assoc Res Otolaryngol 2000;1:33-45.

2) Starr A, Picton TW, Sininger Y, Hood LJ, Berlin CI. Auditory neuropathy. Brain 1996;119(Pt 3):741-53.

3) Sininger Y, Oba S. Patients with auditory neuropathy: who are they and what can they hear? In: Sininger YS, Starr A, editors. Auditory Neuropathy: a new perspective on hearing disorders. Albany, NY: Singular/Thompson Learning;2001. p.15-35.

4) Khimich D, Nouvian R, Pujol R, Tom Dieck S, Egner A, Gundelfinger ED, et al. Hair cell synaptic ribbons are essential for synchronous auditory signalling. Nature 2005;434:889-94.

5) Amatuzzi MG, Northrop C, Liberman MC, Thornton A, Halpin C, Herrmann B, et al. Selective inner hair cell loss in premature infants and cochlea pathological patterns from neonatal intensive care unit autopsies. Arch Otolaryngol Head Neck Surg 2001;127:629-36.

6) El-Badry MM, Ding DL, McFadden SL, Eddins AC. Physiological effects of auditory nerve myelinopathy in chinchillas. Eur J Neurosci 2007;25:1437-46.

7) Buchman CA, Roush PA, Teagle HF, Brown CJ, Zdanski CJ, Grose JH. Auditory neuropathy characteristics in children with cochlear nerve deficiency. Ear Hear 2006;27:399-408.

8) Merchant SN, McKenna MJ, Nadol JB Jr, Kristiansen AG, Tropitzsch A, Lindal S, et al. Temporal bone histopathologic and genetic studies in Mohr-Tranebjaerg syndrome (DFN-1). Otol Neurotol 2001;22:506-11.

9) Ceranić B, Luxon LM. Progressive auditory neuropathy in patients with Leber's hereditary optic neuropathy. J Neurol Neurosurg Psychiatry 2004;75:626-30.

10) Corley VM, Crabbe LS. Auditory neuropathy and a mitochondrial disorder in a child: case study. J Am Acad Audiol 1999;10:484-8.

11) Forli F, Mancuso M, Santoro A, Dotti MT, Siciliano G, Berrettini S. Auditory neuropathy in a patient with mitochondrial myopathy and multiple mtDNA deletions. J Laryngol Otol 2006;120:888-91.

12) Berlin CI, Hood LJ, Morlet T, Wilensky D, Li L, Mattingly KR, et al. Multi-site diagnosis and management of 260 patients with auditory neuropathy/dys-synchrony (auditory neuropathy spectrum disorder). Int J Audiol 2010;49:30-43.

13) Berlin CI, Hood LJ, Goforth-Barter L, Bordelon J. Auditory neu- 
ropathy: three time courses after early identification. ARO Abstract 1999;22:169.

14) Berlin CI, Hood LJ, Morlet T, Den Z, Goforth L. The search for auditory neuropathy patients and connexin 26 patients in schools for the deaf. ARO Abstracts 2000;23:23.

15) Rance G, Cone-Wesson B, Wunderlich J, Dowell R. Speech perception and cortical event related potentials in children with auditory neuropathy. Ear Hear 2002;23:239-53.

16) Rance G, Beer DE, Cone-Wesson B, Shepherd RK, Dowell RC, King AM, et al. Clinical findings for a group of infants and young children with auditory neuropathy. Ear Hear 1999;20:238-52.

17) Kumar AU, Jayaram M. Auditory processing in individuals with auditory neuropathy. Behav Brain Funct 2005;1:21.

18) Starr A, Sininger YS, Pratt $H$. The varieties of auditory neuropathy. J Basic Clin Physiol Pharmacol 2000;11:215-30.

19) Narne VK, Prabhu P, Chandan HS, Deepthi M. Audiological profiling of 198 individuals with auditory neuropathy spectrum disorder. Hearing Balance Commun 2014;12:112-20.

20) Berlin CI, Hood L, Morlet T, Rose K, Brashears S. Auditory neuropathy/dys-synchrony: diagnosis and management. Ment Retard Dev Disabil Res Rev 2003;9:225-31.

21) American National Standards Institute (ANSI). American National Standard Specification for Personal Noise Dosimeters (ANSI S1.251991) (Revision of ANSI S1.25-1978). Melville, NY: American National Standards Institute.

22) Grassi M, Soranzo A. MLP: a MATLAB toolbox for rapid and reliable auditory threshold estimation. Behav Res Methods 2009;41:20-8.

23) Yalçinkaya F, Muluk NB, Ataş A, Keith RW. Random Gap Detection Test and Random Gap Detection Test-Expanded results in children with auditory neuropathy. Int J Pediatr Otorhinolaryngol 2009; 73:1558-63.

24) Green DM. A maximum-likelihood method for estimating thresholds in a yes-no task. J Acoust Soc Am 1993;93(4 Pt 1):2096-105.

25) Martin BA, Stapells DR. Effects of low-pass noise masking on auditory event-related potentials to speech. Ear Hear 2005;26:195-213.

26) Pradeep Y. Identification of sub-groups of subjects with auditory dyssynchrony who may benefit from hearing aids [dissertation]. Bangalore: National Institute of Mental Health \& Neurosciences;2013.

27) Starr A, Picton TW, Kim R. Pathophysiology of auditory neuropathy. In: Sininger Y, Starr A, editors. Auditory Neuropathy: A New Perspective on Hearing Disorders. San Diego, CA: Singular Press;2001. p.67-82.

28) Zeng FG, Oba S, Garde S, Sininger Y, Starr A. Temporal and speech processing deficits in auditory neuropathy. Neuroreport 1999;10: 3429-35.

29) Rapin I, Gravel JS. Auditory neuropathy: a biologically inappropriate label unless acoustic nerve involvement is documented. J Am Acad Audiol 2006;17:147-50.

30) Deltenre P, Mansbach AL, Bozet C, Christiaens F, Barthelemy P, Paulissen D, et al. Auditory neuropathy with preserved cochlear microphonics and secondary loss of otoacoustic emissions. Audiology 1999;38:187-95.

31) Michalewski HJ, Starr A, Nguyen TT, Kong YY, Zeng FG. Auditory temporal processes in normal-hearing individuals and in patients with auditory neuropathy. Clin Neurophysiol 2005;116:669-80.

32) Zeng FG, Kong YY, Michalewski HJ, Starr A. Perceptual consequences of disrupted auditory nerve activity. J Neurophysiol 2005; 93:3050-63.

33) Zeng FG. Speech perception in individuals with auditory neuropathy. J Speech Lang Hear Res 2006;49:367-80.

34) Rance G. Auditory neuropathy/dys-synchrony and its perceptual consequences. Trends Amplif 2005;9:1-43.

35) Rance G, McKay C, Grayden D. Perceptual characterization of children with auditory neuropathy. Ear Hear 2004;25:34-46.

36) Herdman AT, Picton TW, Stapells DR. Place specificity of multiple auditory steady-state responses. J Acoust Soc Am 2002;112:1569-82.

37) Hood LJ. A review of objective methods of evaluating auditory neural pathways. Laryngoscope 1999;109:1745-8.

38) Oates PA, Kurtzberg D, Stapells DR. Effects of sensorineural hearing loss on cortical event-related potential and behavioral measures of speech-sound processing. Ear Hear 2002;23:399-415.

39) Yuvaraj P, Mannarukrishnaiah J. Cortical Evoked Potentials and Hearing Aids in Individuals with Auditory Dys-Synchrony. J Int Adv Otol 2015;11:236-42.

40) Schaub A. Digital Hearing Aids. New York: Thieme Medical Publishers;2008. p.65-77.

41) Rosen S. Temporal information in speech: acoustic, auditory and linguistic aspects. Philos Trans R Soc Lond B Biol Sci 1992;336:36773.

42) Phillips DP, Comeau M, Andrus JN. Auditory temporal gap detection in children with and without auditory processing disorder. J Am Acad Audiol 2010;21:404-8.

43) Javel E. Basic response properties of auditory nerve fibres. In: Altschuler RA, Bobbin RP, Hoffman DP, editors. Neurobiology of hearing: the cochlea. New York: Raven press;1986. p.213-45. 